Chirurg 2015 · 86:1063

DOI 10.1007/s00104-015-0102-0

Online publiziert: 19. Oktober 2015

(c) Springer-Verlag Berlin Heidelberg 2015

CrossMark
P.M. Vogt ${ }^{1} \cdot$ R. Ipaktchi ${ }^{1} \cdot$ B. Weyand ${ }^{1} \cdot$ C. Radtke ${ }^{1} \cdot$ J.K. Krauss ${ }^{3} \cdot$ T. Lenarz $^{2}$

${ }^{1}$ Kliniken und Polikliniken für Plastische, Hand- und Wiederherstellungschirurgie,

Medizinische Hochschule Hannover, Hannover, Deutschland

${ }^{2}$ Hals-Nasen- und Ohrenheilkunde, Medizinische Hochschule Hannover, Hannover, Deutschland

${ }^{3}$ Neurochirurgie, Medizinischen Hochschule Hannover, Hannover, Deutschland

\section{Erratum zu: Weichteilplastische Maßnahmen bei Komplikationen im Schädelbereich}

In diesem Beitrag wurde der Name von Herrn Professor J.K. Krauss versehentlich falsch angegeben. Wir bitten die korrekte Schreibweise zu beachten und entschuldigen den Fehler.

\section{Korrespondenzadresse}

\section{Prof. Dr. P.M. Vogt}

Kliniken und Polikliniken für Plastische, Hand- und Wiederherstellungschirurgie, Medizinische Hochschule Hannover Carl-Neubergstr. 1, 30625 Hannover Vogt.Peter@MH-Hannover.de 